\title{
Step flow breaking during epitaxial growth as a source of surface chemical structuration in functional perovskite thin films
}

\author{
Laura López-Mir, ${ }^{1}$ Lluis Balcells, ${ }^{1}$ S. Valencia, ${ }^{2}$ F. Kronast, ${ }^{2}$ Benjamín Martínez, ${ }^{1}$ Juan José de Miguel $^{3}$ \\ and Carmen Ocal ${ }^{1 *}$ \\ ${ }^{1}$ Institut de Ciència de Materials de Barcelona (ICMAB-CSIC), Campus UAB, 08193 Bellaterra, Barcelona, Spain \\ ${ }^{2}$ Helmholtz-Zentrum-Berlin, Albert-Einstein-Str.15, 12489 Berlin, Germany \\ ${ }^{3}$ Dpt. Física de la Materia Condensada, Condensed Matter Physics Center (IFIMAC) and Instituto de Física de Ma- \\ teriales “Nicolás Cabrera”. Universidad Autónoma de Madrid, Cantoblanco 28049, Madrid, Spain
}

\begin{abstract}
The spontaneous formation of double chemical terminated surfaces of $\mathrm{La}_{0.7} \mathrm{Sr}_{0.3} \mathrm{MnO}_{3}$ due to deviations from step flow during its epitaxial growth on $\mathrm{SrTiO}_{3}(001)$ is presented. The development of surface regions with differentiated topmost composition leads to outstanding surface nano-structuration presenting notably distinct local properties, particularly in terms of electric transport and local work function. Correlation between combined atomic force microscopy and X-ray photoelectron spectroscopy data allows the chemical identification of the two terminations as corresponding to $\mathrm{La}_{0.7} \mathrm{Sr}_{0.3} \mathrm{O}$ and $\mathrm{MnO}_{2}$. The atomistic process at the origin of the surface organization has been interpreted by Monte Carlo simulations that reveal the importance of stacking fault formation as growth proceeds. We present a physical understanding of the experimental results and provide grounds for nanoscale engineering of surfaces in which different electronic responses coexist. The conclusions of the present work can be extended to the growth of other perovskite thin films, therefore broadening the field of functional materials nanostructuration and offering the capability of confining properties at the nanoscale with major interest in a large variety of fields.
\end{abstract}

\section{INTRODUCTION}

The fabrication of epitaxial oxide thin films with well-defined atomically sharp interfaces relies on the successful realization of atomically flat and single-terminated substrate surfaces. On the one hand, it is known that good quality epitaxial oxide thin film growth requires the use of single crystalline substrates with the appropriate lattice parameter. On the other hand, the stoichiometry of the topmost surface plane of the substrate determines the atomic stacking sequence of the deposited films and, therefore, the use of substrates having a unique chemical termination is a critical issue for obtaining sharp hetero-epitaxial interfaces. Nevertheless, increasing interest in these issues arises from the possibility of modulating the interface properties, to control their impact on the films grown on top. Albeit a wise approximation consists 
in using substrates with nanostructured chemical terminations, ${ }^{1,2,3,4,5,6}$ simpler procedures without the need of substrate structuration are desired. In this context, we present a further step towards obtaining tailored 3D architectures in which both chemically sharp (bottom) and modulated (top) interfaces can be integrated within the same fabrication process.

During epitaxial growth on vicinal surfaces, presenting atomic steps due to the substrate miscut, most atoms impinging the surface diffuse long enough to attach to the step edges. In this scenario nucleation of islands on the terraces may be neglected and growth proceeds by step flow. However, ideal step flow may break for specific values of the parameters governing surface evolution (temperature, diffusion length, etc.) provoking growth instabilities. Some of the consequences of such a situation are: i) step meandering leading to terraces of modulated width, ii) island nucleation at the surface terraces if atomic diffusion is reduced and, iii) mound formation in which 3D growth occurs due to a sufficiently inhibited interlayer mass transport. In practice, meandering and islands nucleation develop nearly simultaneously. As instabilities tend to appear as more material is deposited, deviation from the ideal 2D growth occurs easier for increasing thickness complicating the controlled fabrication of thick epitaxial films. Nevertheless, some benefits may result from this phenomenon and getting away from the ideal behavior constitutes a way to obtain spontaneously formed nanostructures leading to modulated local functional properties of thin film surfaces.

Among other perovskites, $\mathrm{La}_{0.7} \mathrm{Sr}_{0.3} \mathrm{MnO}_{3}$ (LSMO) is a widely studied material due to its particular magnetic and electrical properties, where exotic electronic phases emerge from coupling of charge, spin, orbital and lattice degrees of freedom. The perovskite oxide $\mathrm{SrTiO}_{3}$ (STO), with lattice parameter equal to $0.3905 \mathrm{~nm},{ }^{7}$ is the most common substrate for epitaxial growth of complex oxide thin films and constitutes an ideal substrate in the case of LSMO (pseudo-cubic unit cell, u.c., with lattice parameter of 0.389 $\mathrm{nm}$ ) because the resulting lattice mismatch for LSMO//STO is only of 0.39\%. STO(001) single crystals consist of an alternating stacking sequence of atomic layers, $\mathrm{TiO}_{2}$ and $\mathrm{SrO}$ planes ordered in the [001] direction with inter-plane distance of $\approx 0.2 \mathrm{~nm}$ (1/2 u.c.). Therefore, STO has two possible (001) surface termination planes, i.e., SrO and $\mathrm{TiO} 2$. However, it is known that as-received STO(001) surfaces usually contain a coexistence of these two distinct atomic terminations and only after appropriate preparation methods single terminated $\left(\mathrm{SrO}\right.$ or $\left.\mathrm{TiO}_{2}\right)$, ${ }^{8,9}$ or two-terminated self-separated ( $\mathrm{SrO}$ and $\mathrm{TiO} 2$ ) surfaces can be obtained. ${ }^{1}$ LSMO ultra-thin films $(\approx 10 \mathrm{~nm})$ grown onto STO presenting laterally modulated $\mathrm{SrO}^{-\mathrm{TiO}_{2}}$ chemical composition have been shown to grow in a layer by layer (lbl) fashion in which complete LSMO unit cells are piled at each termination. In such conditions, the growing epitaxial film followed the stacking sequence of the underlying substrate and, therefore, two terminated LSMO films were obtained. ${ }^{10}$ In these systems, in which overlayer and substrate have similar crystal structure, the lbl process keeps unchanged the step-terrace structure of the substrate and film formation can be analyzed as a step-flow growth mode. 
In this work, LSMO thin films have been grown on STO substrates using RF magnetron sputtering, a deposition method with high deposition rate and wide range of available coverages, leading to high quality films useful for industrial use. However, given growth parameters (temperature, oxygen pressure...) or even defects (cation excess, oxygen vacancies...) that may be present in the substrate surface can provoke local structural or stoichiometric deviations in the films and in their surfaces. ${ }^{11,12,13}$ Taking advantage of this fact, chemically nanostructured LSMO surfaces can be achieved without the need of special substrate preparation. In order to demonstrate that, thin films of LSMO have been grown on diverse substrates exhibiting either $\mathrm{TiO}_{2}$ or $\mathrm{SrO}$ single chemical termination. The films have been characterized by means of spatially resolved techniques, namely Atomic Force Microscopy (AFM) and X-ray generated photoemission electron microscopy (XPEEM) providing correlation between morphological, compositional and electrical responses. With the same growth conditions, all obtained LSMO films present surfaces with a bimodal distribution in properties typical of surfaces with two different chemical terminations. Thanks to Monte Carlo simulations, this conclusive result is interpreted in terms of the formation of stacking faults in the film during growth.

\section{METHODS}

\section{Experimental}

LSMO films were deposited on $5 \times 5 \times 0.5 \mathrm{~mm}^{3}$ single-crystal STO substrates using RF magnetron sputtering. Atomically flat $\mathrm{TiO}_{2}$-terminated substrates were obtained by ultrasonic consecutive baths in deionized water, acetone and ethanol to selectively etch the SrO layer, followed by annealing up to $1000^{\circ} \mathrm{C}$ for 2 hours in a furnace tube in air for recrystallization. SrO-terminated substrates were prepared by longer annealing of 72 hours at $1300^{\circ} \mathrm{C}^{2}$ LSMO was deposited from stoichiometric targets under a 190 mTorr partial oxygen pressure and at a substrate temperature of $900^{\circ} \mathrm{C}$. After deposition, an in-situ sample annealing at $900^{\circ} \mathrm{C}$ for 60 minutes under an oxygen pressure of 400 Torr was performed followed by cooling down to room temperature (RT) at a rate of $25^{\circ} \mathrm{C} / \mathrm{min}$. All LSMO films investigated here have a nominal thickness of $\approx 18 \mathrm{~nm}$-equivalent to 46 monolayers (ML)- defining the ML as an atomic layer or plane, i.e., one half of the perovskite unit cell.

The morphology and local properties of the samples surfaces were investigated by means of different AFM modes in which in addition to topography a secondary or even a ternary signal can be simultaneously measured. Topography and phase contrast data were obtained by amplitude modulation AFM (AMAFM) in dynamic intermittent-contact. Phase imaging consists in measuring the phase lag (secondary channel) of the cantilever oscillation relative to the external driving oscillation. ${ }^{14}$ This phase shift evolves depending on the tip-surface interactions ${ }^{15}$ therefore reflecting the local surface properties. In particular, it reflects heterogeneities in the composition of the surface because different materials produce different changes in the tip oscillation due to unequal adhesion, friction or viscoelastic forces. ${ }^{16}$ During tip scanning in the AFM contact mode under controlled load, in addition to cantilever normal deflection (topography), 
the cantilever torsion caused by friction can be monitored as a lateral deflection (secondary channel) of the reflected laser beam. This lateral force imaging (also known as Friction Force Microscopy, FFM) is also used to highlight differences in composition of diverse surface regions, ${ }^{17,18}$ simultaneously, the local electrical response (ternary channel) of the surface was explored by conductive AFM (CAFM). These measurements are presented here either in the form of current maps or current versus voltage (I-V) curves taken at selected points on the surface using an MFP3D Asylum AFM equipped with an ORCA module with a specially-designed cantilever holder that encloses two different amplifiers which allow recording currents from the pA to the $\mu \mathrm{A}$ range. In the employed measuring set-up, the conductive tip mounted in the cantilever holder is used as a top electrode and the voltage is applied directly to the sample to establish a current flow through the LSMO film. Probes with different conducting coatings (TiIr and PtSi) mounted on cantilevers with force constants $(\mathrm{k})$ in the range between 2 and $50 \mathrm{~N} / \mathrm{m}$ were used. Applied forces are in the $100 \mathrm{nN}$ range and to minimize any degradation effect due to sample aging, data were taken in asprepared samples.

Space resolved Spectroscopic information has been obtained by means of X-ray Photoelectron emission microscopy (X-PEEM) in two different working modes; (i) collecting the emitted secondary electrons at

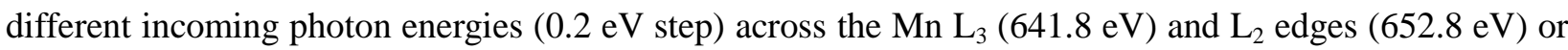
(ii) by collecting photoelectrons at selected kinetic energies (Ek) across the Mn 3p core-level ( $E_{k}=145.8$ $\mathrm{eV})$ for a fixed energy of the incoming photons $\left(\mathrm{E}_{\mathrm{ph}}=200 \mathrm{eV}\right)$. While the former probes the unoccupied Mn 3d levels via dipole-allowed 2p $\rightarrow$ 3d transitions (X-ray absorption spectroscopy, XAS) the later explores the Mn 3p occupied states (X-ray photo electron spectroscopy, XPS).

\section{Monte Carlo simulations}

In order to identify the key parameters controlling the morphology of the growing LSMO films we have performed kinetic, solid-on-solid Monte Carlo simulations in which the STO substrate is represented by a square lattice of $100 \times 150$ sites, with a straight step on one side and periodic boundary conditions in the surface plane. The substrate exhibits a single termination; hence, the step has double layer height, corresponding to 1 u.c. of the STO. The deposition of the incoming material is mimicked by creating monomers at free sites on the surface and allowing them to perform a certain number of diffusion cycles before the arrival of another one.

The system evolution is simulated by using the Metropolis algorithm: at each step a new configuration is generated by displacing a monomer from its current position to a nearest- or next-nearest-neighboring one, and the change in the total energy of the system is computed according to the set of interactions defined. The normalized difference of the relative probabilities of both the initial and final configurations, as measured by their corresponding Boltzmann factors, is compared to a random number between 0 and 1 and chosen accordingly, in such a way that displacements that reduce the system's total energy have a much larger likelihood to succeed than those producing an increase of energy. This process is repeated for 
all monomers susceptible to move on the sample until the specified number of diffusion cycles has been completed and a new deposition event takes place. In order to speed up the simulations and to avoid unphysical situations monomers that are already buried or having a large number of lateral bonds are skipped and therefore not attempted to move during each sweep. Overhangs are not allowed.

\section{RESULTS AND DISCUSSION}

As commented above employing substrates presenting a unique and well defined chemical termination is convenient for obtaining atomically sharp interfaces and good quality layers in thin film growth. Epitaxial growth of LSMO on single terminated STO will proceed such that if the topmost surface of the STO substrate is the $\mathrm{TiO}_{2}$-plane, the created interface will be /.../TiO $/ \mathrm{Ta}_{1-\mathrm{x}} \mathrm{Sr}_{\mathrm{x}} \mathrm{O}$. After deposition of n complete unit cells, the stacking sequence will be /... $/ \mathrm{TiO}_{2} /\left(\mathrm{La}_{1-\mathrm{x}} \mathrm{Sr}_{\mathrm{x}} \mathrm{O} / \mathrm{MnO}_{2}\right)_{\mathrm{n}}$, i.e. the LSMO film will have a $\mathrm{MnO}_{2}$-terminated surface. On the other hand, if the substrate is terminated at the SrO-plane, the stacking sequence will be /.../SrO/(MnO2/ $\left.\mathrm{La}_{1-\mathrm{x}} \mathrm{Sr}_{\mathrm{x}} \mathrm{O}\right) \mathrm{n}$ leading to a $\mathrm{La}_{1-\mathrm{x}} \mathrm{Sr}_{\mathrm{x}} \mathrm{O}$-terminated surface. For an ideal growth, in both cases the global surface should replicate the surface morphological relief (as in step flow growth) and would have laterally homogeneous properties as corresponding to single chemically terminated surfaces. As already mentioned, this is the case for few nanometers thick thin films. ${ }^{1}$ In the present case we will show that for thicker LSMO films (several tens of nanometers) the growth mode can be altered.
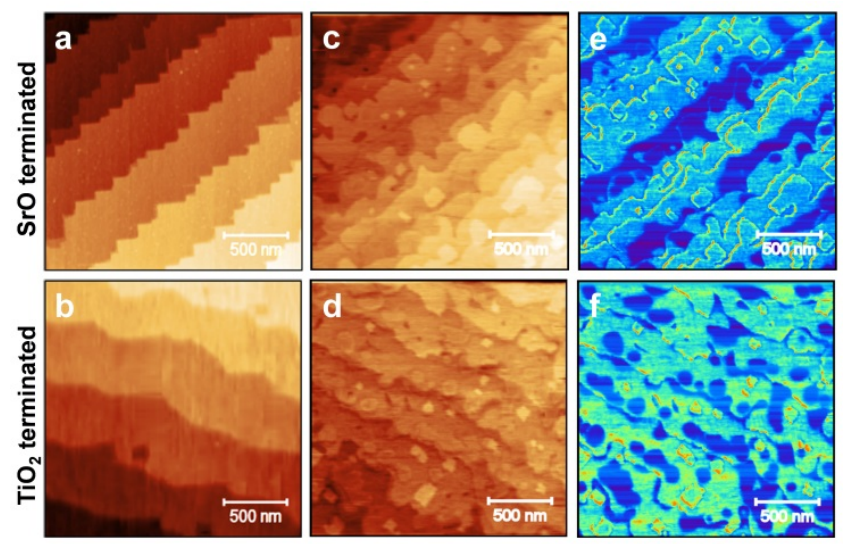

Figure 1. Topographic images of (a) $\mathrm{SrO}$ and (b) $\mathrm{TiO}_{2}$ single terminated $\mathrm{STO}(001)$ substrates, also referred as TO-STO and SO-STO, respectively, in the text. Topography (c, d) and current maps (e, f) of LSMO fims grown respectively on the corresponding substrates. Current maps where obtained with a TiIr tip at $V_{\text {sample }}=1$ V. Difference between low and high currents is $310 \mathrm{nA}(\mathrm{e})$ and $675 \mathrm{nA}(\mathrm{f})$.

In order to explore the influence of substrate termination, we analyze LSMO films of similar thickness $(\approx$ $18 \mathrm{~nm}$ ) grown on different STO substrates. Figure 1 shows results using either films grown on $\mathrm{TiO}_{2}$ or $\mathrm{SrO}$ single terminated STO substrates. The surface morphology of commercially available $\mathrm{TiO}_{2}-$ terminated substrates of STO shows well-defined atomically flat terraces separated by 1 u.c. steps with smooth edges (Figure 1a). The lack of contrast in the corresponding phase-lag image (not shown) con- 
firms that the overall surface exhibits a unique $\mathrm{TiO}_{2}$ termination. Single terminated $\mathrm{SrO}$ substrates can be achieved by annealing during long times $(72 \mathrm{~h})$ at $1300^{\circ} \mathrm{C}{ }^{2}$ The resulting surface consists of atomically flat terraces with step edges facetted along the [100] and [010] directions and step heights of 1 u.c.(Figure 1b).

Along with the data for bare $\mathrm{TiO}_{2}$ - and SrO-terminated substrates (from now on TO-STO and SO-STO, respectively), Figure 1 includes topography (c,d) and current maps (e,f) simultaneously measured for the LSMO films grown on each substrate. After deposition of the LSMO layers, the fairly straight and well defined initial step edges of the initial substrate have disappeared and highly meandered ledges coexist with island nucleation on the terraces. Despite the relatively similar apparent relief of the two LSMO films, in both cases the current readout displays a bimodal electrical response. As a remarkable difference between films, we note that the one grown on the SO-STO substrate (Figure 1c) exhibits fully separated stripes of low and high conducting regions. Even more insightful results the fact of observing small isolated regions of conducting response, the islands height corresponds to $1 \frac{1}{2}$ u.c. In all cases, the step height between terraces with identical conduction coincides with 1 u.c. $(\approx 0.4 \mathrm{~nm})$ while the vertical separation between neighboring levels with different conduction is $1 / 2$ u.c $(\approx 0.2 \mathrm{~nm})$. The first corresponds to the distance between LSMO layers of identical chemical composition (either $\mathrm{La}_{0.7} \mathrm{Sr}_{0.3} \mathrm{O}$ or $\mathrm{MnO}_{2}$ planes) and the second corresponds to the inter-plane distance between consecutive $\mathrm{La}_{0.7} \mathrm{Sr}_{0.3} \mathrm{O}$ and $\mathrm{MnO}_{2}$ atomic layers in LSMO. Indeed, as it has been already reported, ${ }^{10}$ the bimodal response in surface properties is an indication of chemical separation, therefore signaling that the terraces correspond to alternated (001) planes of the LSMO film.

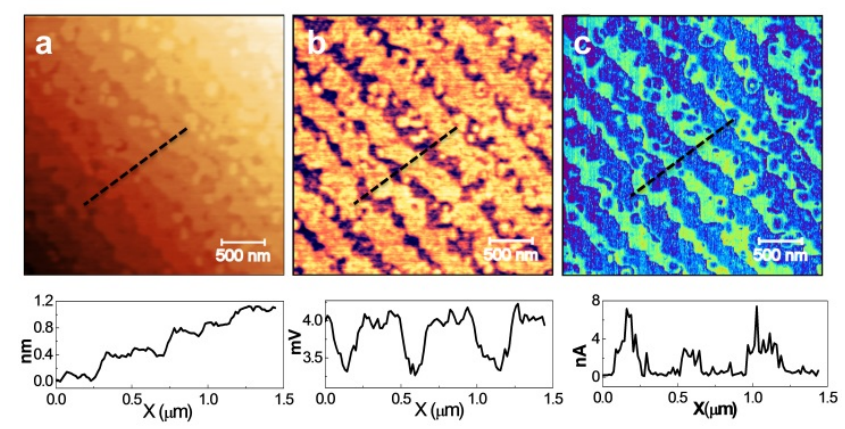

Figure 2. Topography (a), friction (b) and current (c) images obtained in contact $A F M$ at $V_{\text {sample }}=1 \mathrm{~V}$ using a TiIr coated tip. Below each image the corresponding profiles (dashed segments in the images) are shown.

The origin of the bimodal conduction response is cleared up by lateral force imaging (FFM in contact mode) and phase lag imaging (in dynamic AM-AFM) that altogether support the hypothesis of a surface with two chemical terminations. Typical results for two STO substrates with different step density are presented. Figure 2 shows the topography (left), friction force (central) and current (right) maps for a LSMO film grown on a substrate with a miscut angle of $\approx 0.05^{\circ}$ respect to the (001)STO. The local friction signal is defined as half the amplitude of the so-called friction loop, $\mathrm{F}=1 / 2|\mathrm{Fl}(\mathrm{f})-\mathrm{Fl}(\mathrm{b})|$ where $\mathrm{Fl}(\mathrm{i})$ is the lateral 
force signal of the forward $(i=f)$ and the corresponding backward $(i=b)$ scans. Applying this line by line procedure to the complete lateral force images, a friction map is obtained. Consequently, regions of lower friction appear as dark patches in Figure 2b. Comparison of the whole data set confirms a strong correlation and definitively the differentiated regions correspond to areas vertically separated $1 / 2$ u.c. and therefore to planes with different chemical composition.
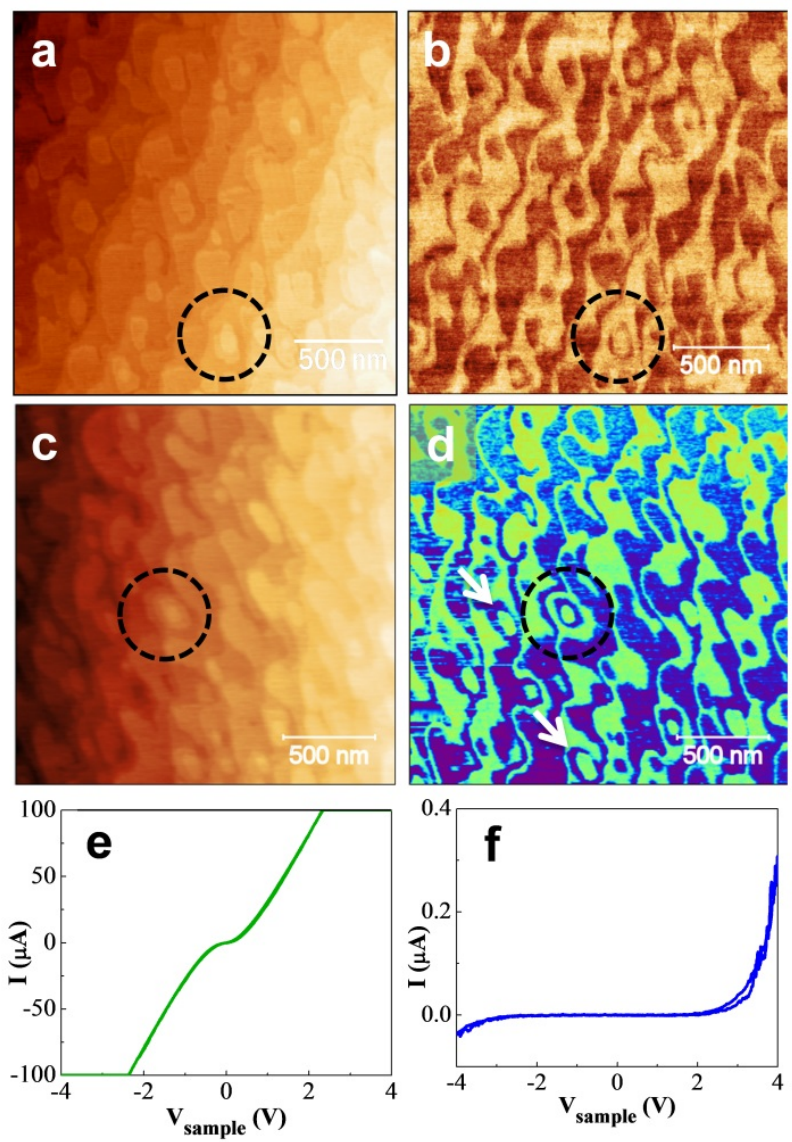

Figure 3. Top: Topography (a) and phase lag (b) images obtained in AM-AFM. Middle: Topography (c) and current map (d) obtained at $\mathrm{V}_{\text {sample }}=2 \mathrm{~V}$ in contact mode AFM using a TiIr coated tip. The difference in current between low and high conducting areas is $18 \mu \mathrm{A}$. Marks on the images are to emphasize the existence of isolated conducting regions: islands (white arrows) and small mounds (encircled). The currentvoltage characteristic corresponds to those taken at diverse regions of the surface with high (e) or low (f) conductivity.

Surface nanostructuration due to the development of regions with different nature is also observed in substrates presenting a slightly larger miscut $\left(\approx 0.09^{\circ}\right)$. Figure 3 shows consecutive AM-AFM (top) and contact AFM (bottom) measurements taken on different surface locations of the same sample. As it can be seen in Fig. 3a-b and 3c-d the same characteristics are observed throughout the whole surface: important step meandering and rounded islands nucleated at terraces. Moreover, incipient mound formation is also noticed (black circles). While the wandering topographic appearance of the steps ledges imaged in Figures 3a and 3c nearly conceals the existence of islands on the narrow terraces, these islands are otherwise clearly distinguished in the secondary channels (white arrows in Figure 3d). Once again, the most notable fact 
results when comparing topography (a, c) with the corresponding phase lag (b) and current (d), respectively. Both phase and current exhibit bimodal signals measured at topographic levels separated by a height equal to $1 / 2$ u.c. $(\approx 0.2 \mathrm{~nm})$. In particular, regions with a diverse phase shift coincide with current differences of about $18 \mu \mathrm{A}\left(\approx 90 \%\right.$ increase at $\left.\mathrm{V}_{\text {sample }}=2 \mathrm{~V}\right)$. Remarkably, this is true even in the case of isolated mounds (encircled in the images) with more than one visible level, because CAFM enables measuring electrical properties at a very local scale, only limiting the measurement to the contact area of the tip that should not be larger than tens of nm2. The existence of surface regions of high conductivity which are laterally isolated has important implications since the properties of perovskite oxide devices can be tuned through interface effects such as spin exchange interactions, ${ }^{19}$ charge transfer, ${ }^{20}$ and band lineups. ${ }^{21}$

Interestingly, a nearly linear I-V characteristics (Figure 3e) is measured at regions with higher conductivity indicating that the local tip-surface system can be viewed as a metal-metal junction in these regions. However, a highly rectifying behavior is observed at the low conducting regions (Figure 3f) suggesting the appearance of a Schottky barrier in the local tip-surface system, which depends on both the work function of the used tip and the local surface work function. The coexistence of two different conducting surface regions indicates that different electronic structures emerge depending on the termination layer, which may have strong relevance for the design of perovskite based devices through modulation of the interfacial Schottky barrier height. ${ }^{22}$ In spite of the diverse general appearance of the LSMO film surfaces presented in Figures 1, 2 and 3, in all cases neighbor terraces with distinct friction, phase contrast and, notably, different read out current would correspond to $\mathrm{La}_{0.7} \mathrm{Sr}_{0.3} \mathrm{O}$ and $\mathrm{MnO}_{2}$ composition.
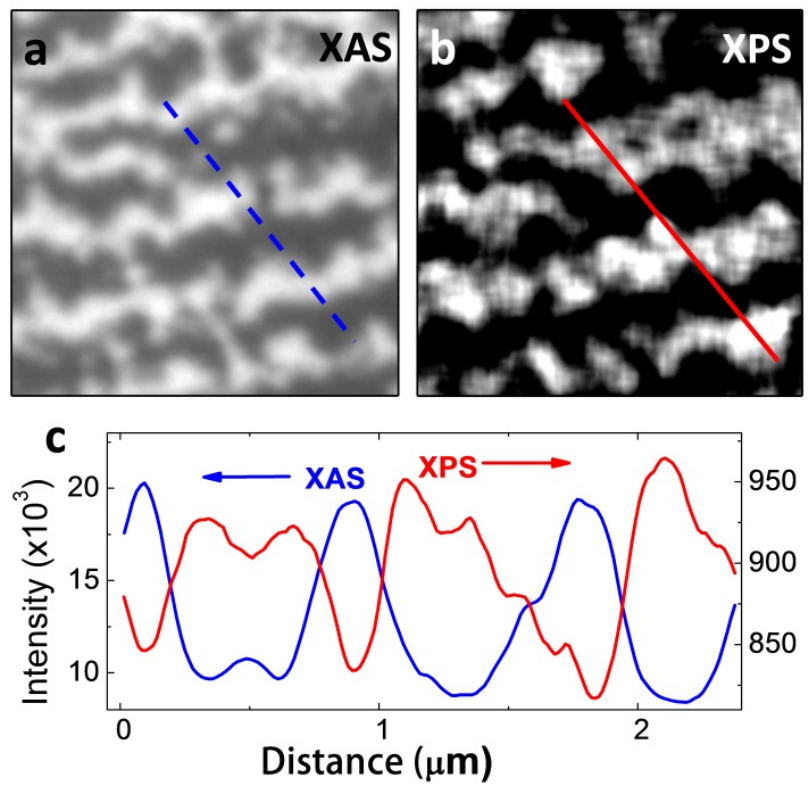

Figure 4. a) XPEEM image working in XAS mode obtained at the pre-edge region of $\mathrm{Mn}_{3,2}$-edges, 630 $\mathrm{eV}$. b) XPS image obtained at the Mn 3p core level $\left(\mathrm{E}_{\mathrm{k}}=145.8 \mathrm{eV}\right)$ and normalized to a pre-edge image $\left(E_{k}=150.0 \mathrm{eV}\right)$. Due to the low counts in the XPS mode a filter has been applied so that each pixel is the average of a 9x9 pixel matrix. c) Profiles marked in (a) and (b). 
To gain a deeper insight into the termination assignation X-PEEM has been used. X-PEEM images obtained in the XAS mode -secondary electrons- show a pattern similar to those described above with two types of domains having different intensity coexist for the whole energy range (630-670 eV) explored (not shown). An example, obtained at $630 \mathrm{eV}$ (pre-edge) is depicted in Figure 4a. Differences in the Mn oxidation state can be excluded as origin of the contrast mechanism since the contrast is present not only at the $\mathrm{Mn} \mathrm{L}_{3,2}$ edges but also at pre- and post-edge spectral regions. Moreover, normalization of the XAS images (see Supporting Information SI1) by that obtained at the pre-edge leads to the loss of contrast between domains for the whole energy range highlighting the fact that the difference in intensity of photo emitted electrons between both regions is just a constant factor (maxima to minima difference in the corresponding profile shown in Figure 4c). The source for the contrast observed by XPEEM may arise from different mechanisms, ${ }^{23,24}$ including topographic features, work function or chemical nature, the latter intimately related. The high resemblance between CAFM (e.g. in Figure 2c) and X-PEEM images suggest a common origin so that the most plausible explanation is that the contrast observed by X-PEEM originates from differences in the conducting character and work function between both regions. Higher work function limits the escape of low energy secondary electrons and will therefore appear darker in XAS images. As proven above, each region is related either to $\mathrm{La}_{0.7} \mathrm{Sr}_{0.3} \mathrm{O}$ or to $\mathrm{MnO}_{2}$ planes, but because the probing depth of the XAS signal is approximately $30 \AA{ }^{25}$ i.e. $\approx 7$ u.c. of LSMO, unambiguous ascription to one or the other termination cannot be made.

To address the chemical composition of the uppermost layer for each surface region we have used energy filtered PEEM (XPS mode) known to be much more surface sensitive than XAS. ${ }^{24,26}$ Figure $4 \mathrm{~b}$ shows an image obtained in this XPS mode for a binding energy $\left(E_{k}=145.8 \mathrm{eV}\right)$ corresponding to the Mn 3p corelevel (see Supporting Information SI2), for which the probing depth is ca. $5 \AA$, i.e. only $\approx 1$ u.c. of LSMO. The image has been normalized by a pre-edge image obtained at $E_{k}=150.0 \mathrm{eV}$. The pre-edge image showed a uniform contrast, i.e. no domains, thus supporting our interpretation of the XAS image contrast as originated from work function differences since topography would also be present in XPS no matter $\mathrm{E}_{\mathrm{k}}{ }^{27}$ The image obtained at the Mn 3p shows a clear binary modulation of contrast, before and after normalization. Since the stacking sequence of $\mathrm{La}_{0.7} \mathrm{Sr}_{0.3} \mathrm{O}$ and $\mathrm{MnO}_{2}$ planes is identical in both regions, higher intensity in XPS arises from regions exhibiting an uppermost $\mathrm{MnO}_{2}$ plane while, in the other regions, the buried $\mathrm{MnO}_{2}$ planes have an intensity attenuated by the $\mathrm{La}_{0.7} \mathrm{Sr}_{0.3} \mathrm{O}$ plane on top. Comparison between line profiles (4c) taken in both XPS (4b) and XAS (4a) images allows correlating the work function information with the atomic termination of the domains. $\mathrm{MnO}_{2}$ planes (bright XPS and dark XAS domains) show higher work function than $\mathrm{La}_{0.7} \mathrm{Sr}_{0.3} \mathrm{O}$ ones (dark XPS and bright XAS domains). The absence of the apical oxygen for the uppermost $\mathrm{MnO}_{2}$ plane would promote electronic segregation leading to i) increase of $\mathrm{Mn}^{3+}$ and ii) insulating properties (large work function). ${ }^{28}$ With all these data at hand and following the previous report including surface potential data in each termination, ${ }^{10}$ planes presenting larger conduction are supposed to be those of $\mathrm{La}_{0.7} \mathrm{Sr}_{0.3} \mathrm{O}$ composition. 
The described results are independent of the initial STO termination and occur for relatively thick films (>40ML) indicating that, contrary to that expected for an ideal layer by layer or step flow growth, they might be interpreted as due to long range instabilities throughout growth. During deposition by RF magnetron sputtering, the different elements that came from the target arrive to the surface and, depending on the growth parameters and substrate characteristics (deposition rate, temperature, substrate miscut, Schowebel barrier...) diffuse until reaching the step edges or nucleating at the terraces. Actually, theoretical studies predict that films over a certain thickness might present instabilities on vicinal surfaces as transitions from step flow to 3D layer-by-layer growth. ${ }^{29}$ As it will be illustrated next by Monte Carlo simulations, our observations cannot be understood in terms of different element diffusion provoking element segregation on the surface but due to staking fault (SF) formation, in which the sequence $\mathrm{La}_{0.7} \mathrm{Sr}_{0.3} \mathrm{O} / \mathrm{MnO}_{2} / \mathrm{La}_{0.7} \mathrm{Sr}_{0.3} \mathrm{O}$ is locally changed to $\mathrm{La}_{0.7} \mathrm{Sr}_{0.3} \mathrm{O} / \mathrm{MnO}_{2} / \mathrm{MnO}_{2}$. Once the SF is formed, further growth maintaining the ideal local stacking leads to local energetically favorable configurations and provides a natural mechanism for the self-organized inhomogeneities over the nanometer scale.

The morphology of the growing LSMO has been simulated by Monte Carlo using the Metropolis algorithm (see methods and Supporting Information). With this type of algorithm the adequate choice of the interactions that describe the system energetics is crucial to obtain the correct outcome. In the simulation we have considered both in-plane nearest-neighbor (nn) and next-nearest-neighbor (nnn) interactions. To account for the existence of the double-layer unit cell and allow for the appearance of the two different surface terminations we have used two types of monomers denoted " $\mathrm{A}$ " and " $\mathrm{B}$ ", representing the $\mathrm{La}_{0.7} \mathrm{Sr}_{0.3} \mathrm{O}$ and $\mathrm{MnO}_{2}$ subunits. The stoichiometry of the growing film is maintained by creating one monomer of each type at each deposition cycle at random positions of the sample. Due to the coexistence of these two species within the growing film, it also becomes necessary to differentiate the lateral interactions depending on the identity of the monomers involved: thus, for $\mathrm{nn}\left(\mathrm{E}_{\mathrm{nn}}^{+,-}\right)$and $\mathrm{nnn}\left(\mathrm{E}_{\mathrm{NNN}}{ }^{+,-}\right)$interactions the upper signs distinguish between attractive $(+)$ or repulsive $(-)$ character depending on the similar (AA, BB) or distinct $(\mathrm{AB})$ nature of the interacting monomers, respectively. Other energetic parameters taken into account are the energy cost of a monomer climbing up a step ( $\left.\mathrm{E}_{\mathrm{up}}\right)$, the energy cost of forming a stacking fault $\left(\mathrm{E}_{\mathrm{SF}}\right)$ and the Ehrlich-Schwoebel barrier $\left(\mathrm{E}_{\mathrm{ES}}\right)$. The complete description of all parameters is summarized in the Supporting Information (Figure SI3) and their values in Table 1. The stacking fault energy $\left(E_{\mathrm{SF}}\right)$ can be loosely related to the cohesive energy of the alternating atomic planes of the LSMO lattice, and constitutes the most distinctive aspect of these simulations, giving rise to the appearance of some unusual behavior when compared to single component systems. A full account of the results of these simulations is nevertheless beyond the scope of this paper and will be presented elsewhere. Here we concentrate on getting some insight into the phenomena that result in the experimentally observed surface morphologies and hence surface properties. We have treated this energy as a fixed parameter (equal to 1) and referred all other interactions to it. Temperature is also given in units of Boltzmann's constant $\mathrm{k}_{\mathrm{B}}$. 

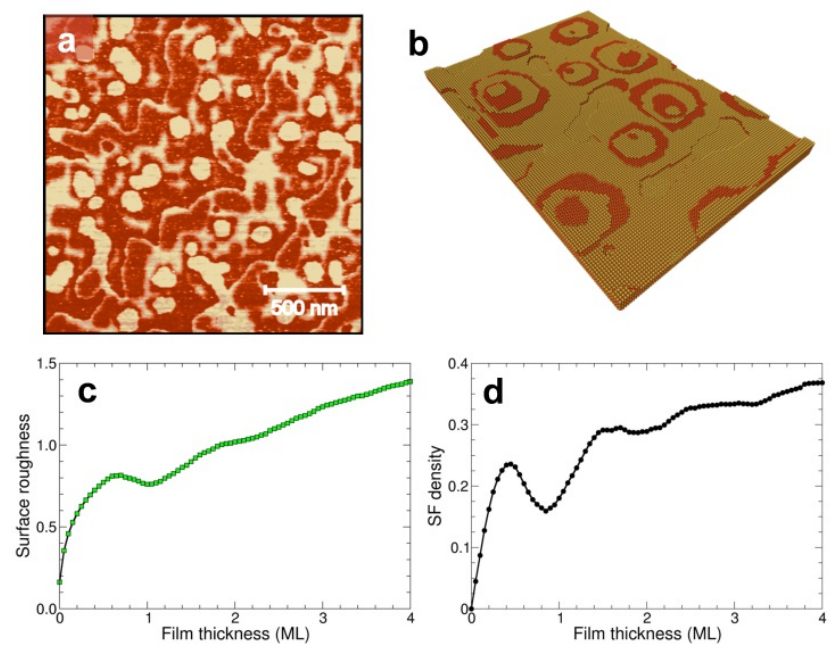

Figure 5. (a) Experimental CAFM map $\left(\mathrm{V}_{\text {sample }}=1 \mathrm{~V}\right)$ showing the bimodal current characteristic and (b) Monte Carlo simulation carried out with the parameters listed in Table 1. (c) and (d) Evolution of the surface roughness and the density of stacking faults with increasing thickness for the film simulated in (b).

In Figure 5 a typical current map obtained by CAFM is compared with the result of a MC simulation using the parameters listed in Table 1; a movie showing the evolution of the simulated growth front during the deposition of several monolayers under these conditions can be found in the Supplementary Information. One can immediately notice the qualitative agreement between experiment and simulation. The main feature is the appearance of islands and mounds exhibiting both terminations (dark and bright areas). The graphs in Figs. 5c and 5d reveal that both the surface roughness and the density of stacking faults steadily increase. As commented, this latter magnitude is of particular interest: since the grown film is stoichiometric, a certain amount of stacking faults must appear in order to accommodate the material that is not completing the double-layer unit cells, thus leading to the appearance of the two terminations. The evolution of the stacking fault density shows oscillations with monolayer periodicity superimposed to the overall increasing trend due to the formation of separated islands of types "A" and "B" with $1 / 2$ u.c. height right after completion of a previous monolayer, when the terraces are wider. As growth continues more stable one u.c. high islands grow laterally, those faulted islands are squeezed between them and a part of this material is forced to climb up to complete higher levels thus reducing the total amount of stacking faults present in the film. Nevertheless, the process is not completed due to kinetic limitations caused by the low temperature, and some faulted patches are left behind becoming buried with subsequent growth.

\begin{tabular}{|c|c|c|c|c|c|c|c|}
\hline $\mathbf{E}_{\mathbf{n n}}{ }^{+} / \mathbf{E}_{\mathbf{S F}}$ & $\mathbf{E}_{\mathbf{n n}}{ }^{-} / \mathbf{E}_{\mathbf{S F}}$ & $\mathbf{E}_{\mathbf{n n n}}{ }^{+} / \mathbf{E}_{\mathbf{S F}}$ & $\mathbf{E}_{\mathbf{n n n}}{ }^{-} / \mathbf{E}_{\mathbf{S F}}$ & $\mathbf{E}_{\mathrm{up}} / \mathbf{E}_{\mathbf{S F}}$ & $\mathbf{E}_{\mathrm{ES}} / \mathbf{E}_{\mathbf{S F}}$ & $\mathbf{T}$ & $\mathbf{N}_{\text {jumps }}$ \\
\hline 1.25 & -0.5 & 0.6 & -0.25 & -0.25 & 0.0 & 0.25 & 250 \\
\hline
\end{tabular}

Table 1. Parameters used in the simulation presented in Fig. 5. Negative energies are repulsive. See Supporting Information. 
The simulation also shows how the initially straight step gradually acquires a meandering morphology. This phenomenon appears as a result of two different causes. In the first place, it is due to the limited diffusion of monomers along the step edge, provoked by the reduced temperature compared to the intensity of the attractive lateral interactions. And secondly, the advancing step overruns and absorbs some of the one u.c. high islands previously formed on the terrace close to the edge. Advancing steps are also known to develop roughness as a result of the so-called "Bales-Zangwill instability" ${ }^{30}$ which appears when there is an Ehrlich-Schwoebel barrier reducing or preventing the incorporation of monomers from the upper side of the step. In this work we have chosen to keep this energy $\mathrm{E}_{\mathrm{ES}}$ equal to zero in order to highlight the other effects that are more specific to this system. One should remark that, since surface diffusion is a thermally activated process, a relatively low temperature such as the one used in the simulation in Figure 5 typically results in short displacements of the deposited monomers. However, these results have been obtained using a rather long diffusion length of 250 jumps per cycle -to be compared to the terrace width of 150 sites. Experimentally, achieving these conditions would require reducing the deposition rate so as to give the deposited monomers more time to move across the surface.

To summarize the conclusions gained from the Monte Carlo simulations, Figure 6 shows the results obtained in a series of limiting cases that would correspond to very different experimental conditions: a) High temperature and high monomer mobility, b) high temperature $(\mathrm{T}=1.0)$, low monomer mobility $(\mathrm{N}=$ 1), c) Low temperature and high monomer mobility $(\mathrm{N}=250)$ and $\mathrm{d})$ low temperature and low monomer mobility $(\mathrm{N}=1)$. A complete description of the surface evolution in each case can be found in the Supporting Information.
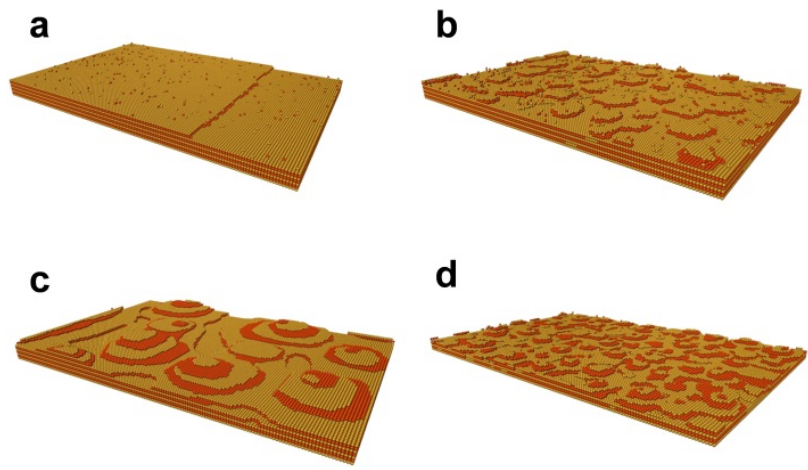

Figure 6. Monte Carlo simulated topographies representative for different limiting conditions: (a) High temperature $(\mathrm{T}=1.0)$ and long monomer diffusivity $(\mathrm{N}=250)$. (b) High temperature $(\mathrm{T}=1.0)$ and low mobility $(\mathrm{N}=1)$. (c) Low temperature $(\mathrm{T}=0.25)$ and high monomer mobility $(\mathrm{N}=250)$. (d) Low temperature $(\mathrm{T}=0.25)$ and mobility $(\mathrm{N}=1)$.

For high temperature and high monomer mobility, islands formation is prevented and step flow with smooth edges takes place (Figure 6a). Same mobility and low temperature correspond to the case discussed above and compared in Figure 5 with the experimental results. A further illustration of the typical film morphology obtained in this case is given in Figure 6c. As already commented, its most distinctive 
characteristic is the appearance of patches with both surface terminations. The island sizes are large as corresponds to the long average diffusive length. A lower mobility (shorter diffusive length) maintaining the low temperature will cause the nucleation of many small islands (Figure 6d) avoiding step advancing, increasing roughness but still presenting two terminations.

The results of these simulations allow us to identify the reduced temperature as the key parameter responsible for the formation of the stacking faults that in the end result in the appearance of the two surface terminations of the film. Due to the random nature of the deposition and to the attractive lateral interactions between monomers of the same type, the nucleation of faulted islands is statistically unavoidable. In order to maintain the single termination, those islands must be forced to break up and the resulting monomers have to climb up on top of the non-faulted islands to maintain the correct stacking sequence. When the temperature is low compared to the strength of the lateral bonds of the monomers within the islands, breaking the faulted ones becomes difficult and they are soon stabilized by the growth of layers on their top.

\section{CONCLUSIONS}

The presented results show that the nano-scale structuration of the LSMO surface is consequence of chemical self-organization and is caused by deviation of local stoichiometry developed within an energy landscape during growth. Whereas in layer by layer growth similar chemical nanostructuration originates from the replica of patterned substrates, here and based on Monte Carlo simulations the formation of stacking faults during growth is proposed to be at the origin of the experimental observations. Both situations offer different ways for engineering frameworks of metal and insulator two dimensional domains. Remarkably, unambiguous chemical identification of the two terminations $\left(\mathrm{La}_{0.7} \mathrm{Sr}_{0.3} \mathrm{O}\right.$ or $\left.\mathrm{MnO}_{2}\right)$ presenting a different work function, has been possible by X-ray spectroscopy analysis. Because the domain formation is self-sustained, external stimuli provoking instabilities during growth (defects, oxygen pressure, temperature...) can be used to sensitively manipulate the size distribution of coexisting metallic and insulating regions, thus making practical the control of nano-engineered functional domains in perovskite thin films. In particular the capability of confining properties at the nanoscale is of major interest for many challenging applications (e.g. confined electron gas, confined chemical reactions...). The presented results demonstrate that long range order control could appear as a simple way to create new functionalities at the surface, particularly, the formation of local electronic nanostructures.

\section{ASSOCIATED CONTENT}

Supporting Information. Space resolved Spectroscopic information (Mn L 3,2 and Mn 3p spectra) and simulations details including the complete list of interactions used in the simulations. This material is available free of charge via the Internet at http://pubs.acs.org. 
AUTHOR INFORMATION

Corresponding Author

Carmen Ocal

*E-mail: cocal@icmab.es

\section{ACKNOWLEDGMENT}

This work has been supported by the Spanish Government under projects MAT2013-47869-C4 and MAT2015-71664-R. The ICMAB members acknowledge the Spanish MINECO through the project MAT2015-68994-REDC and the Severo Ochoa Program (SEV-2015-0496), FEDER program and the Generalitat de Catalunya through 2014 SGR 501. L. LM thanks the Spanish MINECO for a FPI BES2013-063424 fellowship. JJM thanks the Centro de Computación Científica (CCC) of the Universidad Autónoma de Madrid for computing time.

\section{REFERENCES}

(1) Sánchez, F; Ocal, C.; Fontcuberta, J. Tailored surfaces of perovskite oxide substrates for conducted growth of thin films. Chem. Soc. Rev., 2014, 43, 2272-2285.

(2) Bachelet, R.; Sanchez, F.; Palomares, F.J.; Ocal, C.; Fontcuberta, F. Atomically flat SrO-terminated $\mathrm{SrTiO}_{3}(001)$ substrate. Appl. Phys. Lett. 2009, 95(14):141915 (1-3).

(3) Kleibeuker, J. E.; Koster, G.; Siemons, W.; Dubbink, D.; Kuiper, B.; Blok, J. L.; Yang, C. H.; Ravichandran, J.; Ramesh, R.; Ten Elshof, J. E.; Blank, D. H. A.; Rijnders, G. Atomically Defined Rare-Earth Scandate Crystal Surfaces. Adv. Funct. Mater. 2010, 20, 3490-3496.

(4) Bachelet, R.; Ocal, C.; Garzón, L.; Fontcuberta, J.; Sánchez, F. Conducted growth of $\mathrm{SrRuO}_{3}$ nanodot arrays on self-ordered $\mathrm{La}_{0.18} \mathrm{Sr}_{0.82} \mathrm{Al}_{0.59} \mathrm{Ta}_{0.41} \mathrm{O}_{3}(001)$ surfaces. Appl. Phys. Lett. 2011, 99, 051914 (1-3).

(5) Bachelet, R.; Sánchez, F.; Santiso, J.; Munuera, C.; Ocal, C.; Fontcuberta, J. Self-Assembly of $\mathrm{SrTiO}_{3}(001)$ Chemical-Terminations: A Route for Oxide-Nanostructure Fabrication by Selective Growth. Chem. Mater. 2009, 21, 2494-2498.

(6) Paradinas, M.; Garzón, L.; Sánchez, F.; Bachelet, R.; Amabilino, D. B.; Fontcuberta, J.; Ocal, C. Tuning the local frictional and electrostatic responses of nanostructured $\mathrm{SrTiO}_{3}$ surfaces by self-assembled molecular monolayers. Phys. Chem. Chem. Phys. 2010, 12, 4452-4458.

(7) Ruddlesden, S.N.; Popper, P.; New compounds of the $\mathrm{K}_{2} \mathrm{NIF}_{4}$ type Acta Crystallogr. 1957, 10, 538-539.

(8) Kawasaki, M.; Takahashi, K.; Maeda, T.; Tsuchiya, R.; Shinohara, M.; Ishiyama, O.; Yonezawa, T.; Yoshimoto, M.; Koinuma, H. Atomic Control of the $\mathrm{SrTiO}_{3}$ Crystal Surface. Science 1994, 266, 1540 1542. 
(9) Koster, G.; Kropman, B. L.; Rijnders, G. J.; Blank, H. M.; Rogalla, D. H. A. Quasi-ideal strontium titanate crystal surfaces through formation of strontium hydroxide. Appl. Phys. Lett. 1998, 73, 2920-2922.

(10) Ocal, C.; Bachelet, R.; Garzón, L.; Stengel, M.; Sánchez, F.; Fontcuberta, J. Nanoscale Laterally Modulated Properties of Oxide Ultrathin Films by Substrate Termination Replica through Layer-by-Layer Growth. Chem. Mater. 2012, 24, 4177-4184.

(11) Tselev, A.; Vasudevan, R.K.; Gianfrancesco, A.G.; Liang Qiao, L.; Ganesh, P.; Meyer, T.L.; Lee, H.N.; Biegalski, M.D.; Baddorf, A.P.; Kalinin, S.V. Surface control of epitaxial manganite films via oxygen pressure. ACS Nano, 2015, 4, 4316-4327

(12) Li , Z.; Bosman, M.; Yang, Z.; Ren, P.; Wang, L.; Cao, L.; Yu , X.; Ke, Ch.; Breese , M-B.H.; Rusydi, A.; Zhu, W.; Dong, Z.; Foo, Y.L. Interface and surface cation stoichiometry modified by oxygen vacancies in epitaxial manganite films. Adv. Funct. Mater., 2012, 22, 4312-4321

(13) Shimizu, R.; Ohsawa, T.; Iwaya, K.; Shiraki, S.; Hitosugi, T. Epitaxial growth process of $\mathrm{La}_{0.7} \mathrm{Ca}_{0.3} \mathrm{MnO}_{3}$ thin films on $\mathrm{SrTiO}_{3}(001)$ : thickness-dependent inhomogeneity caused by excess $\mathrm{Ti}$ atoms. Crys. Growth Des. 2014, 14, 1555-1560.

(14) Schirmeisen, A.; Anczykowski, B. ; Fuchs, H. Dynamic force microscopy. In Springer Handbook of Nanotechnology. Springer Berlin Heidelberg, 2004, pages 449-473.

(15) Chen, X.; Roberts, C.J.; Zhang, J.; Davies, M.C.; Tendler, S.J.B. Phase contrast and attraction repulsion transition in tapping mode atomic force microscopy. Surf. Sci., 2002, 519, 593-598.

(16) Barth, C.; Foster, A. S.; Henry, C. R.; Shluger, A. L. Recent Trends in Surface Characterization and Chemistry with High-Resolution Scanning Force Methods. Adv. Mater. 2011, 23, 477-501.

(17) Frisbie, C. D.; Rozsnyai, L. F.; Noy, A.; Wrighton, M. S.; Lieber, C. M. Functional group imaging by chemical force microscopy. Science, 1994, 265, 2071-2074.

(18) Munuera, C.; Shekhah, O.; Wang, H.; Wöll, Ch.; Ocal, C. The controlled growth of oriented metalorganic frameworks on functionalized surfaces as followed by scanning force microscopy. Phys. Chem. Chem. Phys., 2008, 10, 7257-7261.

(19) Izumi, M.; Ogimoto, Y.; Okimoto, Y.; Manako, T.; Ahmet, P.; Nakajima, K.; Chikyow, T.; Kawasaki, M.; Tokura, Y. Insulator-metal transition induced by interlayer coupling in $\mathrm{La}_{0.6} \mathrm{Sr}_{0.4} \mathrm{MnO}_{3} / \mathrm{SrTiO}_{3}$ superlattices. Phys. Rev. B, 2001, 64, 064429 (1-6).

(20) Chakhalian, J.; Freeland, J. W.; Habermeier, H.-U.; Cristiani, G.; Khaliullin, G.; van Veenendaal, M.; Keimer, B. Orbital reconstruction and covalent bonding at an oxide interface. Science, 2007, 318, 1114-1117.

(21) Hikita, Y.; Nishikawa, M.; Yajima, T.; Hwang, H.Y. Termination control of the interface dipole in $\mathrm{La}_{0.7} \mathrm{Sr}_{0.3} \mathrm{MnO}_{3} / \mathrm{Nb}: \mathrm{SrTiO}_{3}(001)$ Schottky junctions. Phys. Rev. B, 2009, 79, 073101 (1-4).

(22) Minohara, M.; Yasuhara, R.; Kumigashira, H.; Oshima, M. Termination layer dependence of Schottky barrier height for $\mathrm{La}_{0.6} \mathrm{Sr}_{0.4} \mathrm{MnO}_{3} / \mathrm{Nb}: \mathrm{SrTiO}_{3}$ heterojunctions. Phys. Rev. B, 2010, 81, 235322 (1-6). 
(23) Schneider, C.M.; Schönhense, G. Investigating surface magnetism by means of photoexcitation electron emission microscopy. Rep. Prog. Phys., 2002, 65, R1785-R1789.

(24) Aballe, L.; Matencio, S.; Foerster, M.; Barrena, E.; Sánchez, F.; Fontcuberta, J.; Ocal, C.. Instability

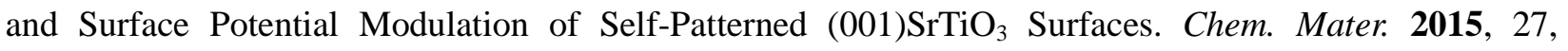
$6198-6204$.

(25) Ruosi, A.; Raisch, C.; Verna, A.; Werner, R.; Davidson, B.A.; Fujii, J.; Kleiner, R.; Koelle, D. Electron sampling depth and saturation effects in perovskite films investigated by soft x-ray absorption spectroscopy. Phys. Rev. B , 2014, 60, 125120 (1-8).

(26) Seah, M.P.; Dench, W.A. Quantitative electron spectroscopy of surfaces: A standard data base for electron inelastic mean free paths in solids. Surf. Interf. Anal., 1979, 1, 2-11.

(27) Workfunction contrast is only expected to appear at kinetic energies around the photoemission threshold.

(28) Calderón, M.J.; Brey, L.; Guinea, F. Surface electronic structure and magnetic properties of doped manganites. Phys. Rev. B, 1999, 60, 6698-66704.

(29) Beausoleil, A.; Desjardins, P. ; Rochefort, A. Impact of nucleation on step-meandering instabilities during step flow growth on vicinal surfaces. Phys. Rev. E, 2014, 89(3):032406 (1-14).

(30) Bales, G.S.; Zangwill, A. Morphological instability of a terrace edge during step-flow growth. Phys. Rev. B, 1990, 41, 5500-5508. 


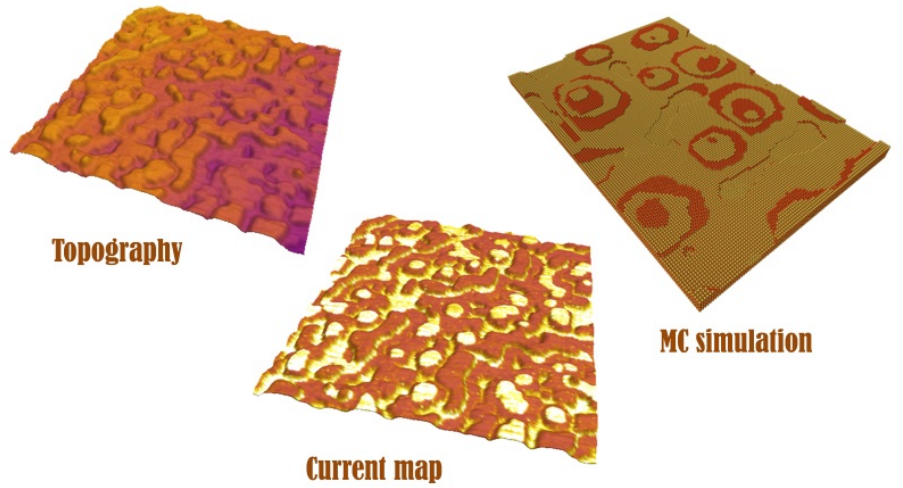

